\title{
Driver's Awareness and Lane Changing Maneuver in Traffic Flow based on Cellular Automaton Model
}

\author{
Kohei Arai ${ }^{1}$ \\ 1 Graduate School of Science and Engineering \\ Saga University \\ Saga City, Japan
}

\author{
Steven Ray Sentinuwo ${ }^{2}$ \\ 2 Sam Ratulangi University, \\ Kampus Unsrat, Manado, Indonesia
}

\begin{abstract}
Effect of driver's awareness (e.g., to estimate the speed and arrival time of another vehicle) on the lane changing maneuver is discussed. "Scope awareness" is defined as the visibility which is required for the driver to make a visual perception about road condition and the speed of vehicle that appears in the target lane for lane changing in the road. Cellular automaton based simulation model is created and applied to simulation studies for driver awareness behavior. This study clarifies relations between the lane changing behavior and the scope awareness parameter that reflects driver behavior. Simulation results show that the proposed model is valid for investigation of the important features of lane changing maneuver.
\end{abstract}

Keywords-traffic cellular automata; scope awareness; lane changing maneuver; driver perception; speed estimation

\section{INTRODUCTION}

Recent study on the traffic flow reports that the traffic congestion is influenced not only by the road capacity condition, but also by the driver behavior [1]. The other studies also found the strong relationship between the driver' speed behavior and accidents [2]-[6]. There are two separate components which affect human factors in driving, driving skills and driving style [7]. Driving style has a direct relation to the individual drivers' behavior. The U.S. Department of Transportation recently reported that driver behavior is leading to lane-change crashes and near-crashes [8]. In some countries, the reckless driving behaviors such as sudden-stop by publicbuses, tailgating, or vehicles which changing lane too quickly also could give an impact to the traffic flow.

The lane changing maneuver is one of the actions in the highway. Lane changing is defined as a driving maneuver that moves a vehicle laterally from one lane to another lane where both lanes have the same direction of travel. Lane changing maneuvers are occasionally performed in order to avoid hazards, obstacles, vehicle collision, or pass through the slow vehicle ahead. Lane changing requires high attention and visual perceptions compared to normal highway of freeway driving due to the need to continually monitor areas around the subject vehicle [9]. However, in the real traffic situations, there are some reckless drivers that change the lanes at the moment when they signal or who make "last-minutes-decision" on the road. Frequent lane changing in roadway could affect traffic flow and even lead accidents. The lane changing behaviors can be very depended on the characteristic of the driver [10].

There are some crashes of accidents typically referred to as Look-But-Fail-To-See errors because drivers involved in these accidents frequently. These are reported that they failed notification of the conflicting vehicles in spite of looking in the appropriate directions; commonly occur when drivers change lanes [25]. This means that the drivers typically use their visual perception in order to estimate the speed and the arrival time of the other vehicles before making a maneuver, e.g., lane changing maneuver.

A psychology study is also reported that the accuracy level of this visual perception may lead to both failures, detect the collision and judge the crash risk (e.g., time-to-contact). From a certain distance, a short fixation may be enough to identify an approaching vehicle. If gaze duration for stimulus processing is long, then it is complicated processing while it is short for simple processing. Inaccuracy of the gazes' duration is likely to reflect a failure to process these stimuli [26].

The Cellular Automata model of Nagel and Schreckenberg (NaSch) [12] is improved for showing effects of scope awareness that reflect drivers' behavior when they are making a lane changing. This NaSch model has been modified to describe more realistic movement of individual vehicle when make a lane changing maneuver. Moreover, the recent study of spontaneous braking behavior [1] enhances the driver's scope awareness behavior. The proposed model is based on NaSch model which takes into account scope of awareness and spontaneous braking in order to clarify the effects of these drivers' behavior.

This paper is organized as follows. Section 2 presents a theoretical aspect of traffic CA model. Section 3 explains the proposed model. Section 4 describes simulation process and the results in the form of fundamental diagrams and space-time diagrams. Finally, in section 5, a summary and conclusion is described with some discussions.

\section{TRAFFIC Cellular Automata Model}

One of the famous microscopic models for road traffic flow simulation is Cellular Automata (CA) model. CA model is a discrete computability mathematical model. In comparison with another microscopic model, CA model based approach is efficient [11] and is used for dynamic system simulations. CA model consists of two components, a cellular space and a set of state. The state of a cell is completely determined by its nearest neighboring cells.

All neighborhood cells have the same size in the lattice. Each cell can either be empty, or is occupied by exactly one node (car in this simulation model). There is a set of local transition rule that is applied to each cell from one discrete 
time step to another (i.e., iteration of the system). This parallel updating from local simple interaction leads to the emergence of global complex behavior. Furthermore, the utilization of CA model successfully explains the phenomenon of transportation. These traffic cellular automata (TCA) are dynamical systems that are discrete in nature and powerful to capture all previously mentioned basic phenomena that occur in traffic flows [11].

The one dimensional cellular automata model for single lane freeway traffic is introduced by Nagel and Schreckenberg (NaSch) [12]. This model shows how traffic congestion can be thought of as an emergent or collective phenomenon due to interactions between cars on the road, when the car density is high (cars are close to each on average). According to NaSch model, the randomization rule captures natural speed fluctuations due to human behavior or varying external conditions [14].

In a real traffic situation, most highways have two or more lanes. Regarding this road condition, there are a few analytical models for multi-lane traffic. Nagatani is one of the first researchers who introduced a $\mathrm{CA}$ model for two lane traffic [24]. Then, in addition to the Nagatani's model, Rickert et al. [15] considers a model with $v_{\max } \geq 1$. Their model introduces the lane changing behavior for two lanes traffic. They proposed a symmetric rule set where the vehicle changes lanes if the following criteria are fulfilled:

$\operatorname{gap}(\mathrm{i})<1$

$\operatorname{gap}_{0}(\mathrm{i})>1_{0}$

$\operatorname{gap}_{0, \text { back }}(\mathrm{i})>1_{0, \text { back }}$

The variable $\operatorname{gap}(i), \operatorname{gap}_{0}(\mathrm{i})$, and $\operatorname{gap}_{0, \text { back }}(\mathrm{i})$ denote the number of empty cells between the vehicle and its predecessor on its current lane, and forward gap on the desired lane, and backward gap on the desired lane, respectively. Rickert also uses the parameters which allow decide how far the vehicle look ahead in the current lane for $l$, ahead on the desired lane for $l_{0}$, and how far the vehicle look back on the desired lane for $l_{0, \text { back }}$.

The advanced analysis about lane-changing behavior is reported which includes symmetric and asymmetric rules of lane-changing [16]-[19], [20]-[23].

\section{PROPOSED MOdEL DEFINITION AND SiMUlation}

The proposed model uses two-lane highway with unidirectional traffic character in periodic boundaries condition. Two-lane model is necessary in order to accommodate the lane changing behavior in the real traffic condition. A onedimensional chain of $\mathrm{L}$ cells of length $7.5 \mathrm{~m}$ represents each lane. This value is considered as the length of vehicle plus the distance between vehicles in a stopped position. A one-lane consists of $10^{3}$ cells. There are just two possible states of each cell. Each cell can only be empty or containing by just one vehicle. The speed of each vehicle is integer value between $v=$ $0,1, \ldots, v_{\max }$. In this model, all vehicles are considered as homogeneous and have the same maximum speed $v_{\max }=5$. The speed value number corresponds to the number of cell that the vehicle proceeds at one time step. The state of a road cell at the next time step, form $t$ to $t+1$ is dependent on the states of the direct frontal neighborhood cell of the vehicle and the core cell itself of the vehicle.

Rickert et al. [15] discusses criteria of safety by introducing the parameters which decide how far the vehicle looks ahead on current lane, looks ahead on desired lane, and looks back on desired lane. Those criteria have to be fulfilled before a vehicle makes a lane changing. However, in real traffic condition, these criteria of safety rules by Rickert are not sufficient to describe driver's behaviors in highway traffic. This paper introduces a new additional parameter to accommodate the driver behavior when making a lane changing. In addition to considering the gap of cell that consists of vehicle, the speed parameter of the other vehicle that situated in the desired lane is taken into account. The parameter of scope awareness $S_{a}$ that reflects the various characters of driver is introduced. The scope awareness parameter represents drivers' behavior when lane changing. $S_{a}$ value reflects the degree of driver aggressiveness and awareness. Fig.1 shows schematic definition diagram of scope awareness $S_{a}$ from the perspective of the vehicle (the third cell on lane 2) in its current speed and position $v_{(1) ;} x_{(1)}$

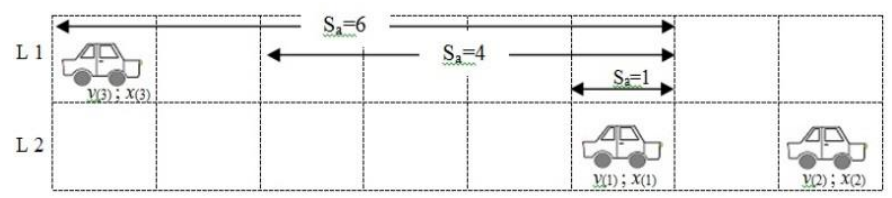

Fig. 1. Schematic definition diagram of scope awareness $S_{a}$ from the perspective of the vehicle (the third cell on lane 2) in its current speed and position $v_{(1) ;} x_{(1)}$

$S_{a}=1$ implies very dangerous because the driver of vehicle lintend to make a lane changing even the gap between the car and the car ahead of the car on the next lane is only one cell. On the other hand, the lane changing can be done safely when $S_{a}=6$ because the gap between both is six cells.

In contrast to the gap-length model's parameter [15], the scope awareness parameter $S_{a}$ accommodates the sight distance taken by the driver to make a perception about road-lane condition and the speed of other vehicles that exist in target lane. Therefore, the subject vehicle would consider changing its lane not only due to the comparison value between the number of gap and condition which decide how far the vehicle look ahead in the current lane, but also depending on the current speed of the subject vehicle and the speed of other vehicles that situated along the scope awareness area.

The updating rule for lane changing maneuver is done in accordance with a set of rules. In comparison to the lane changing model of Rickert et al. [15], there are two basic differences rules from the proposed model. The first one, as the result of traffic conditions ahead of subject driver, the subject vehicle would consider changing its lane not only due to the comparison value between the number of gap and condition which decide how far the vehicle look ahead in the current lane, but also depending on the current speed of the subject vehicle that can be varied based on traffic situation. Another difference is the scope awareness value $\left(S_{a}\right)$. The subject vehicle would consider the speed of vehicles that situated along with its scope 
awareness area then decide whether possible or not to change the lane.

At the beginning of iterations, the subject driver checks desirability of a lane changing. The subject driver looks ahead to check if the existing gap in the current lane can accommodate the current speed. If not, then due to the randomness number of percentage ratio, the subject driver decides whether the driver maintains or decelerates the vehicle speed due to the existing gap number or change the lane. When the subject driver chooses to "lane changing", then driver looks sideways at the other lane to check whether the cell next to the subject vehicle is empty and the forward gap on the other lane is equal or longer than the current lane. If one cell is unoccupied or free-cell then its state is 0 . Moreover, the subject driver also looks back at the other lane to check road condition. In real traffic situations, a subject driver also has to look back on the other lane in order to estimate the velocity of the following vehicle to avoid a collision. Equation (8) accommodates the driver behavior that estimate the velocity of vehicle at the moment before making a lane changing.

If there is another vehicle within the area of scope awareness, then the subject driver estimates the speed of the vehicle in order to avoid collision during the lane changing maneuver. The subject driver makes a lane changing maneuver if the speed of the vehicle that is located within the area of scope awareness is less than the existing gap. The lane changing rules can be summarized as follows:

gap $_{\text {same }}<v_{\text {current }}$

cell $_{\text {next }}=0$

$\operatorname{rand}()<\mathrm{p}_{\text {change }}$

gap $_{\text {target }}>$ gap $_{\text {same }}$

$\mathrm{v}_{\text {Vehicle,back }} \leq$ gap $_{\text {back }} ; \mathrm{X}\left(\right.$ vehicle $\left._{\text {back }}\right) \in \mathrm{S}_{\mathrm{a}}$

The lane changing rules are applied to the vehicle that changes from right lane to left lane and conversely. The vehicle only moves sideways and it does not advance. Once all the lane changing maneuvers are made, then the updating rules from a single lane model are applied independently to each lane. Fig.2 shows the schematic diagram of lane changing operation.

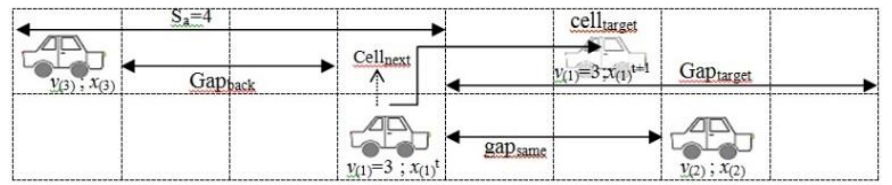

Fig. 2. Schematic diagram of a lane changing operation

In Fig.2, the subject vehicle $v_{(1)} ; x_{(1)}$ is assumed that have the current speed $v_{(1)}{ }^{t}=3$ cell per time step and the parameter of scope awareness $S_{a}=4$ cells. In order to avoid the introduction of any unrealistic artifacts in the simulation then the proposed model uses eq. (7) to express the more realistic lane changing decision. According to eq. (7), the driver must consider that the forward gap in the desired lane is more than the gap in the current lane. This consideration is important because the proposed model uses the different desired velocities for the vehicles.
Once the lane changing maneuvers are made to all possible vehicles, then the updating rules from a single lane model are applied independently to each lane. Together with a set of lane changing rules, the road state is obtained by applying the following rules to all by parallel updated:

Acceleration: $v(\mathrm{i}) \rightarrow \min \left(\mathrm{v}(\mathrm{i})+1, \mathrm{v}_{\max }\right)$

Deceleration: $v(i) \rightarrow \min \left(v(i), \operatorname{gap}_{\text {same }}(i)\right)$

Driving: $x(i) \rightarrow x(i)+v(i)$

\section{SimUlation RESUltS}

The simulation starts with an initial configuration of $N$ vehicles, with fixed distributions of positions on both lanes. The simulation uses the same initial velocity for all vehicle $v_{\text {min }}$ $=0$ and the maximum vehicle speed has been set to $\mathrm{v}_{\max }=5$ cell/time-step. The velocity corresponds to the number of cells that a vehicle advances in iteration. Many simulations are done with the different density $\rho$. The density $\rho$ is defined as the number of vehicles $N$ along with the highway over the number of cells on the highway $L$.

The traffic model uses close (periodic) boundary conditions. This means that during one simulation, the total number of vehicles on the highway cannot be changed. Vehicles move from left to right. If a vehicle arrives on the right boundary then it moves to the left boundary. Since the proposed model assumes symmetry character of the both lanes, the traffic flow characteristics on both lanes are identical.

\section{A. Traffic Flow}

In order to examine the effect of scope awareness on the traffic flow, then the proposed model is simulated over 1000 iterations on $10^{3}$ cells for all possible density level. The flow indicates the number of moving vehicles per unit of time. Along with the study of the proposed model, a comparative study between with and without using scope awareness parameter.

Fig.3 shows the average flow-density diagram of the proposed model is compared to a two-lane traffic system without using scope awareness parameter.

The following results are made:

1) The proposed model reproduces a recognizable diagram of flow towards density relationship. Flow is linearly increasing together with the increases in density level. A maximum flow level is achieved at density level $\rho=0.5$ for each value of $S_{a}$. After reaches the critical point of flow at $\rho=0.5$, the flow at each level of $S_{a}$ becomes linearly decreasing in density. In other words, the laminar flow turn into back travelling start-stop waves after density level $\rho=0.5$.

2) Compared to the model without scope awareness consideration (Fig.3-bottom diagram), the usage of $S_{a}$ parameter produced a better flow of vehicles, especially above density $\rho=0.4$. The Sa parameter maintained the traffic to keep flowing by carefully calculate the appropriate time to make a lane changing decision, thus the lane changing maneuver does not disturb the traffic in the target lane. 


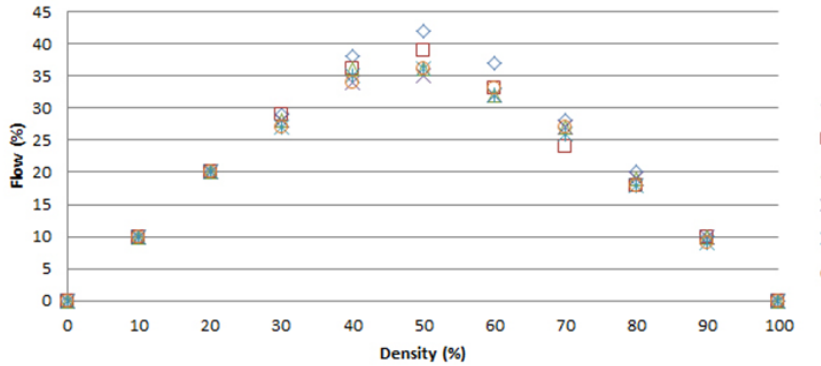

(a) Average flow-density diagram of the proposed model

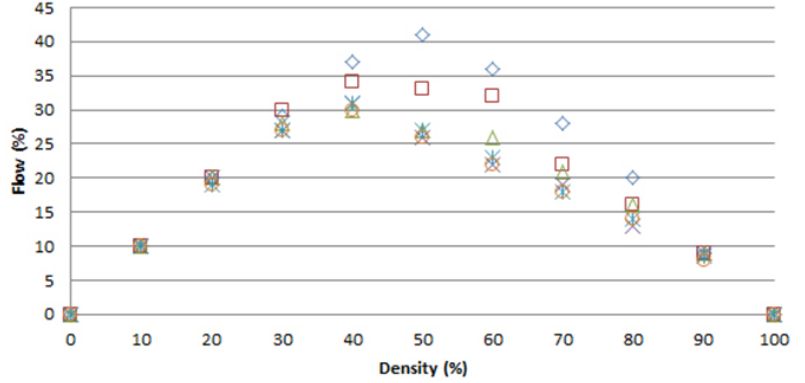

(b) Two-lane traffic system without using scope awareness parameter

Fig. 3. The average flow-density diagram of the proposed model is compared to a two-lane traffic system without using scope awareness parameter

\section{B. Space-Time Diagram}

The space-time diagram represents the location of the vehicles at the certain time. The space-time diagram for density $\rho=0.25, \rho=0.5$, and $\rho=0.75$ is shown. These three values of density assumed as the light traffic, moderate traffic, and heavy traffic in the real traffic condition, respectively. Fig.4 shows the result for density $\rho=0.25$ at all the values of scope awareness while the lane changing probability is $100 \%$. Fig.4 (a) for Scope awareness $\mathrm{Sa}=1$; (b) for Scope awareness $\mathrm{Sa}=2$; (c) for Scope awareness $\mathrm{Sa}=3$; (d) for Scope awareness $\mathrm{Sa}=4$; (e) for Scope awareness $\mathrm{Sa}=5$; (f) for Scope awareness $\mathrm{Sa}=6$, respectively. The horizontal axis represents space while the vertical axis represents the time. Vehicles move from left to right in space axis while from top to bottom in time axis.

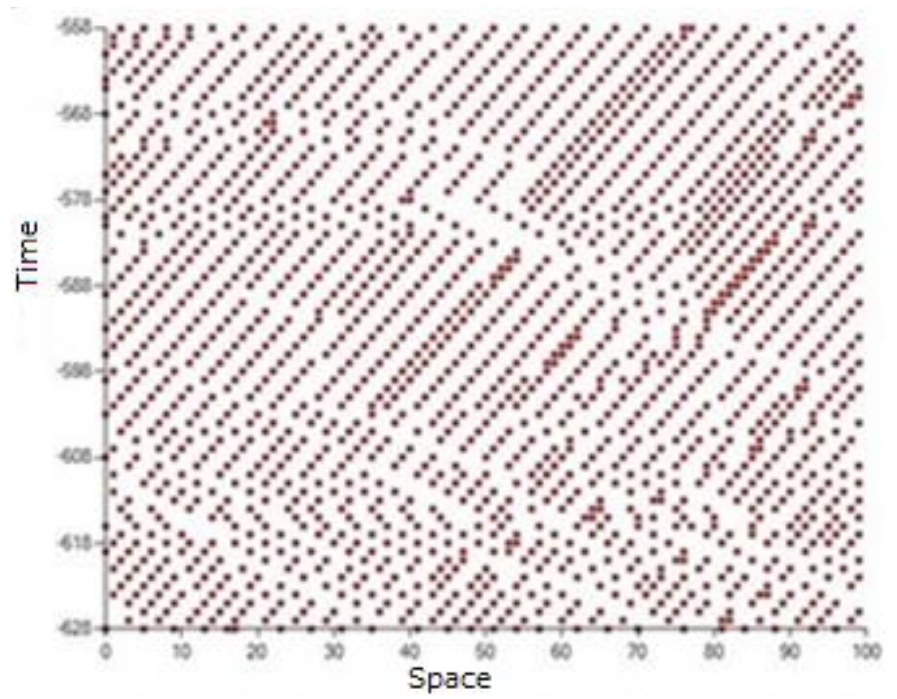

(a) $\mathrm{Sa}=1$



(b) $\mathrm{Sa}=2$

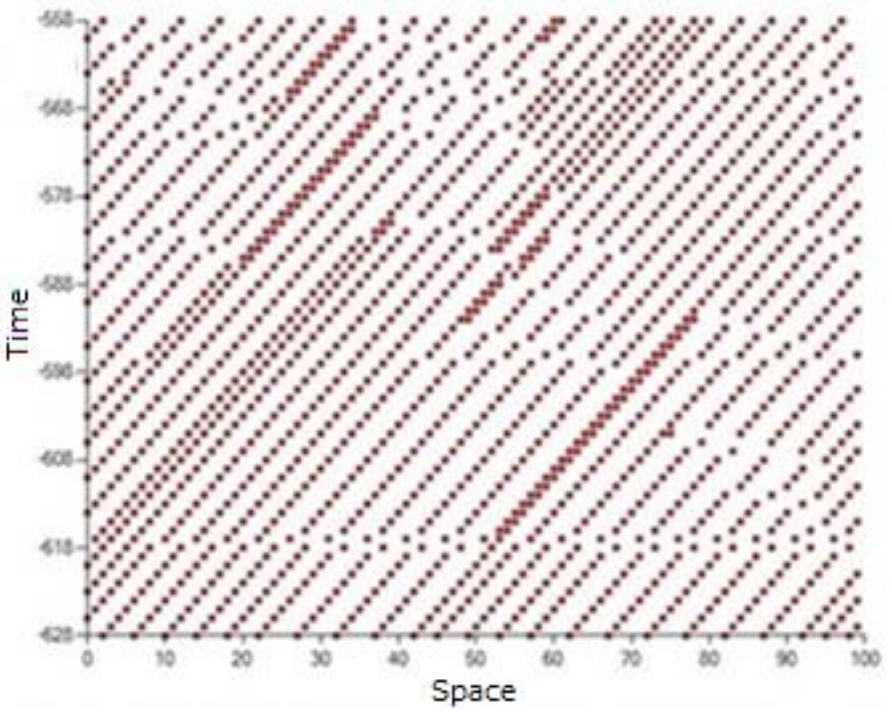

(c) $\mathrm{Sa}=3$

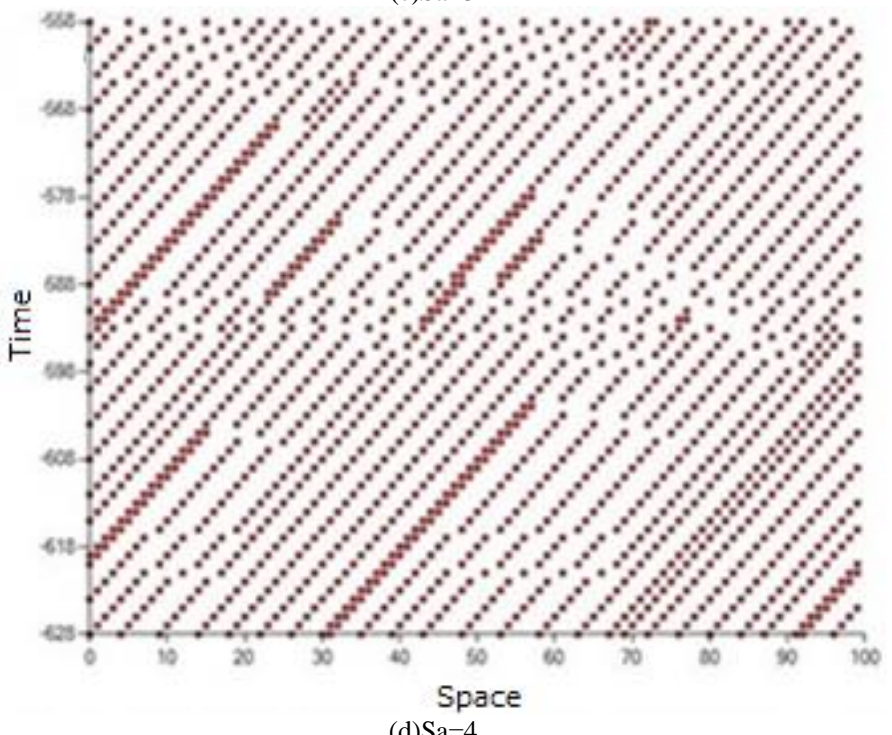

(d) $\mathrm{Sa}=4$ 


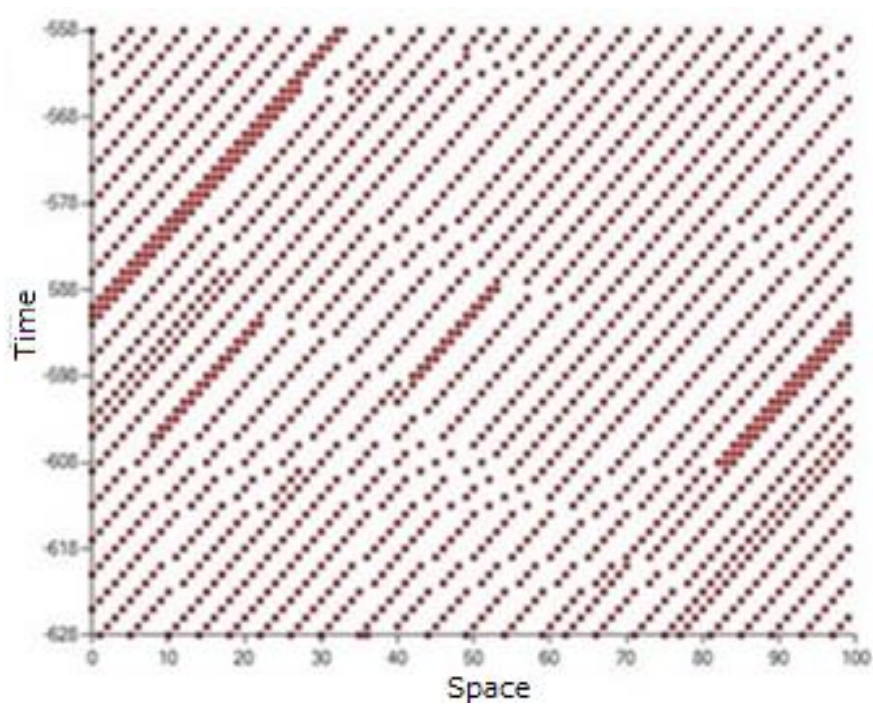

(e) $\mathrm{Sa}=5$

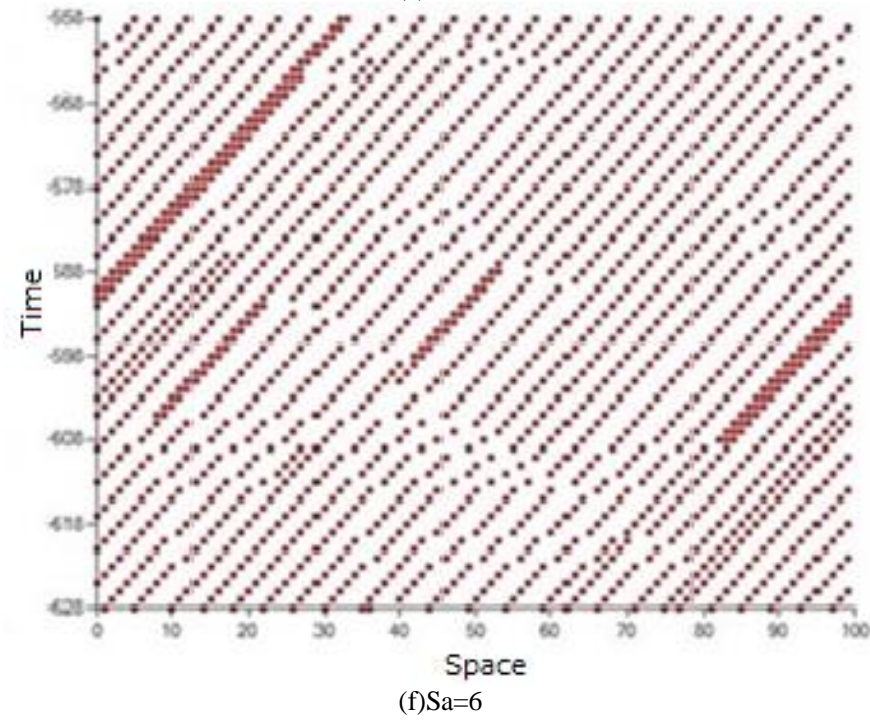

Fig. 4. Space-time diagram for light traffic condition (density $\rho=25 \%$ ). Lane changing probability $100 \%$. (a) for Scope awareness $\mathrm{Sa}=1$; (b) for Scope awareness $\mathrm{Sa}=2$; (c) for Scope awareness $\mathrm{Sa}=3$; (d) for Scope awareness $\mathrm{Sa}=4$; (e) for Scope awareness $\mathrm{Sa}=5$; (f) for Scope awareness $\mathrm{Sa}=6$

In the light traffic condition $\rho=0.25$, the increases of scope awareness distance affect the vehicles flow. Free flow phase showed in $\mathrm{Sa}=1$ diagram (Fig.4 (a)), which are drawn as light area and have shallow negative inclinations. However, when the $\mathrm{Sa}$ value is increased then some solid area appears. The solid area with steep positive inclination reflects the traffic jam. Short-vehicle-life-lines frequently appear and disappear in this case. This implies that there are a great number of lane changing at this traffic density $\rho=0.25$. In accordance with increasing of the scope awareness, occurrence of the shortvehicle-life-lines becomes smaller than before (Fig.4 (f)). Meanwhile, in the heavy traffic condition, traffic situation is independent on scope awareness. In this traffic condition, lane changing is very rare. The result implies that in the heavy traffic condition, drivers' lane changing style have no influence to the traffic situation.
Table 1 (a) and (b) present the ratio of the spontaneous braking number over lane changing by using the scope awareness parameter (SA) and without using the scope awareness parameter, respectively. " 0 " means that the spontaneous braking did not happen, although the lane changing maneuver is still occurring in this certain traffic condition. Meanwhile, "N/A" means that the lane changing maneuver did not occur at all in this traffic condition.

TABLE I. RATIO OF THE SPONTANEOUS BRAKING NUMBER OVER LANE CHANGING WITH THE SCOPE AWARENESS PARAMETER (SA) AND WITHOUT THE SCOPE AWARENESS PARAMETER

\begin{tabular}{|l|l|l|l|l|l|l|}
\multicolumn{7}{|c|}{$(\mathrm{A}) \mathrm{WITH}$ SA } \\
\hline \multirow{2}{*}{ DENSITY $(\%)$} & $\begin{array}{l}\text { SA- } \\
1(\%)\end{array}$ & $\begin{array}{l}\text { SA- } \\
2(\%)\end{array}$ & $\begin{array}{l}\text { SA- } \\
3(\%)\end{array}$ & $\begin{array}{l}\text { SA- } \\
4(\%)\end{array}$ & $\begin{array}{l}\text { SA- } \\
5(\%)\end{array}$ & $\begin{array}{l}\text { SA- } \\
6(\%)\end{array}$ \\
\hline 10 & 56 & 40 & 23 & 24 & 20 & 0 \\
\hline 20 & 82 & 74 & 63 & 38 & 18 & 3 \\
\hline 30 & 81 & 60 & 12 & 0 & 0 & 0 \\
\hline 40 & 82 & 35 & 3 & 0 & 0 & 0 \\
\hline 50 & 92 & 11 & 0 & 0 & 0 & 0 \\
\hline 60 & 83 & 8 & 0 & 0 & 0 & 0 \\
\hline 70 & 85 & 9 & 0 & 0 & 0 & 0 \\
\hline 80 & 80 & 0 & 0 & 0 & 0 & 0 \\
\hline 90 & 88 & 0 & 0 & 0 & 0 & 0 \\
\hline
\end{tabular}

\begin{tabular}{|l|l|l|l|l|l|l|}
\hline \multicolumn{7}{|c|}{ (B)WITHOUT SA } \\
\hline DENSITY(\%) & $\begin{array}{l}\text { SA- } \\
1(\%)\end{array}$ & $\begin{array}{l}\text { SA- } \\
2(\%)\end{array}$ & $\begin{array}{l}\text { SA- } \\
3(\%)\end{array}$ & $\begin{array}{l}\text { SA- } \\
4(\%)\end{array}$ & $\begin{array}{l}\text { SA- } \\
5(\%)\end{array}$ & $\begin{array}{l}\text { SA- } \\
6(\%)\end{array}$ \\
\hline 10 & 53 & 45 & 36 & 48 & 13 & 0 \\
\hline 20 & 82 & 83 & 80 & 63 & 17 & 6 \\
\hline 30 & 71 & 64 & 55 & 9 & 0 & 0 \\
\hline 40 & 74 & 63 & 6 & 0 & 0 & N/A \\
\hline 50 & 85 & 33 & 0 & 0 & N/A & N/A \\
\hline 60 & 89 & 14 & 0 & N/A & N/A & N/A \\
\hline 70 & 91 & 20 & 0 & N/A & N/A & N/A \\
\hline 80 & 100 & 0 & 0 & N/A & N/A & N/A \\
\hline 90 & 100 & N/A & N/A & N/A & N/A & N/A \\
\hline
\end{tabular}

It is found that the ratio is getting large in accordance with density while the ratio is getting small according to SA parameters. Also, the ratio of the case with SA is smaller than that without SA. These implies that the number of spontaneous braking can be suppressed by considering scope of awareness.

\section{Vehicle Speed EstimationEerror}

When the drivers make mistakes in speed estimation by visual perception, then the drivers fails their lane changing. Therefore speed estimation error is a key factor of lane changing. Fig.5 shows the effect of speed estimation error to the lane changing (a) and to the spontaneous braking action (b) in the light traffic $\rho=0.25$, moderate traffic $\rho=0.5$, and heavy traffic $\rho=0.75$, respectively. Fig.5 (c) shows the ratio between both of lane changing and spontaneous braking.

It is obvious that the number of lane changing and spontaneous braking are increased with increasing of the speed estimation error. Also, the number of lane changing and spontaneous braking in light traffic condition is larger than that in moderate and heavy traffic conditions. The reason for this is that it is getting hard for lane changing and spontaneous braking when the traffic condition is getting heavy. Also, it may say that it is getting hard for lane changing and spontaneous braking when the speed estimation error is increased. 


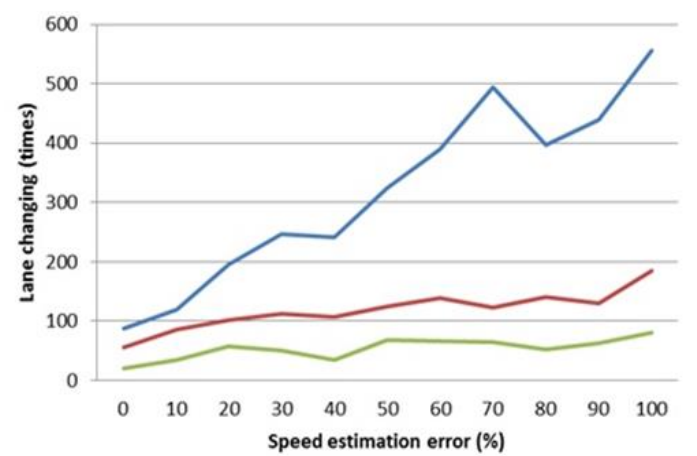

(a)Lane changing manoeuvre

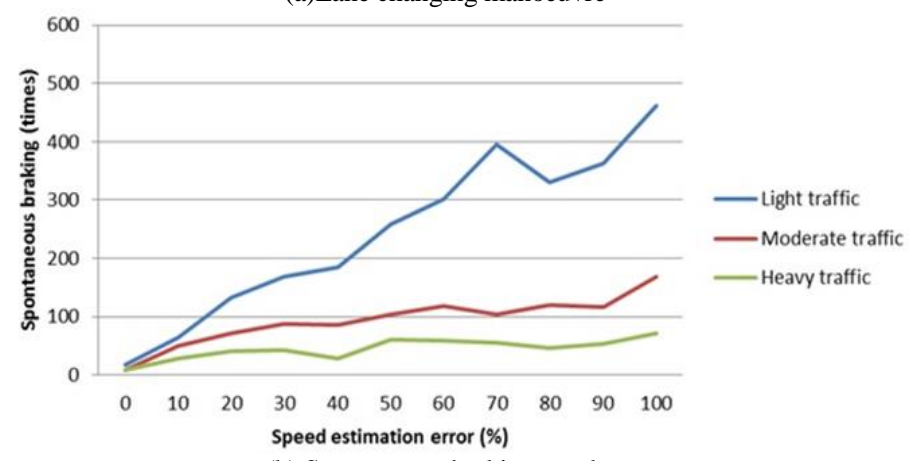

(b) Spontaneous braking number

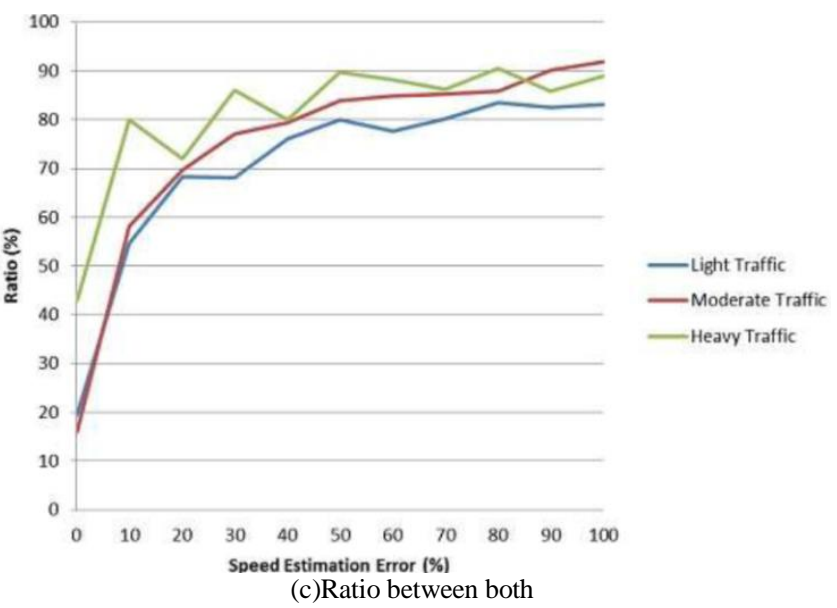

Fig. 5. Effect of speed estimation error to the lane changing manoeuvre (a) and to the spontaneous braking number (b) together with the ratio between both (c). These diagrams are simulated for the case $S_{a}=6$

Lane changing is much influencing to traffic situation than spontaneous braking, in general. Speed estimation error in lane changing is much influencing than that in spontaneous braking. Fig.6 shows Space-Time diagrams with consideration of speed estimation errors in the visual perception based driver's vehicle speed estimation raged from 0 to 1 for the light traffic condition $(\mathrm{SA}=6)$.
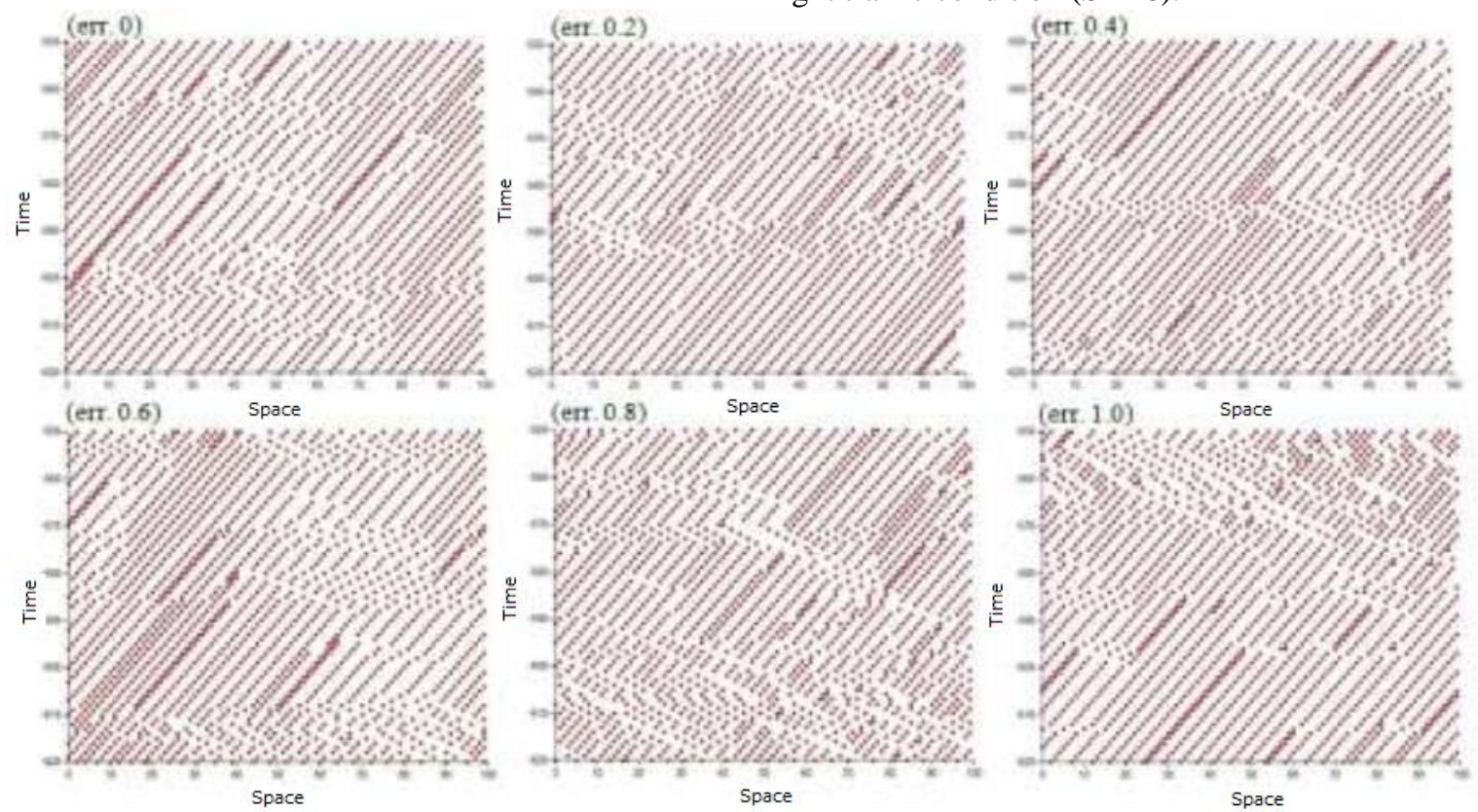

Fig. 6. Space-Time diagrams with consideration of speed estimation errors in the visual perception based driver's vehicle speed estimation raged from 0 to 1 for the light traffic condition $(\mathrm{SA}=6)$

Through a comparison between Fig.4 and 6, it is found that traffic congestions seem to be independent to the speed estimation error and are happened occasionally. The phenomena of short-thin solid lines and wide solid lines also appear in these values of traffic density. The appearance of short-thin solid lines and the disappearance of wide solid line confirmed the conclusion that the short-thin solid line caused by the lane changing maneuver of another vehicle from adjacent lane so the subject vehicle has to make a spontaneous braking in order to avoid collision, and the wide solid line appeared as a result of deceleration into the minimum speed of the vehicle as the consequence of the reduced opportunities for lane changing maneuver.

\section{SUMMARY AND CONCLUSION}

The simulation model of the traffic cellular automata for representation of a driver behavior in a two lane highway is proposed. The term of scope awareness introduced to reflect 
the certain area of roadway that is considered by the driver to make a perception of road condition. This perception includes the estimation speed of vehicles that are located within the scope-awareness distance prior to make a lane changing maneuver. The relation between flow-density and space-time are investigated in order to examine the effect of scope awareness parameter in the traffic flow. The followings are concluded through this study,

1) This model describes the realistic traffic situation, in particular for capturing the situation when driver make a lane changing maneuver. Compared to the conventional approach, the usage of scope awareness model approach produces a better flow of vehicles.

2) The various parameters of the scope awareness may represent the characteristic and the experience level of the drivers. The increases of the scope awareness value means the driver become much aware to estimate the road condition in a lane changing maneuver.

3) The proposed model reveals the phenomena of the short-thin solid line jam and the wide solid line jam in the traffic flow. It is found that the short-thin solid lines are the result of the lane changing maneuver of another vehicle from adjacent lane which makes the subject vehicle which has to make a spontaneous braking in order to avoid collision. As the result of this spontaneous braking, it is causing the other following vehicles which have to adjust or decrease their speed with the vehicle ahead. This action introduces a short queue of vehicles. On the other hand, a wide solid line appears as a result of the deceleration vehicle speed to the minimum speed of the vehicles as the consequence of the reduced lane changing chances. The chance reduction introduces a transient bottleneck effect.

4) The simulation results show that lane changing maneuvers with taking into account another vehicle speed could reduce the level of traffic congestion. However, in the heavy traffic (high dense) situation, the chance of a lane changing is small which results in traffic congestions.

The simulation results show that when the driver become more aware during lane changing decision, then the traffic flow seems to behave a single lane traffic character. In the situation, the vehicles tend to decrease its speed rather than making a lane changing. Moreover, the results indicate that in the density level less than $75 \%$, traffic congestion is reduced by managing of the driver behavior. Other than that, the management of driver behavior makes a significant impact. The simulation result can serve as a reference for transportation planning, evaluation, and control. Moreover, the result paves the way for accurate simulation of a more complex traffic system. Based on the result, the effect of road shape towards the vehicle deceleration is to be studied hereafter.

\section{REFERENCES}

[1] K. Arai and S. Sentinuwo, "Spontaneous-braking and lane-changing effect on traffic congestion using cellular automata model applied to the two lane traffic", (IJACSA) International Journal of Advanced Computer Science and Applications, Vol. 3 (8), 2012.

[2] Elvik, R., Vaa, T. (2004) The Handbook of Road Safety Measures. Elsevier Science, Oxford.
[3] Finch, D. J., Kompfner, P., Lockwood, C. R. \& Maycock, G. (1994) Speed, speed limits and crashes. Project Record S211G/RB/Project Report PR 58. Transport Research Laboratory TRL, Crowthorne, Berkshire.

[4] Nilsson, G. (2004). Traffic safety dimensions and the power model to describe the effect of speed on safety. Lund Bulletin 221. Lund Institute of Technology, Lund.

[5] Salusjärvi, M., 1981. The speed limit experiments on public roads in Finland. Technical Research Centre of Finland. Publication 7/1981. Espoo, Finland.

[6] Lewin, I. (1982). Driver training a perceptual-motor skill approach. Ergonomics, 25, 917-924.

[7] Elander, J., West, R., \& French, D. (1993). Behavioral correlates of individual differences in road traffic crash risk: An examination of methods and findings. Psychological Bulletin, 113, 279-294.

[8] G. M. Fitch, S. E. Lee, S. Klauer, J. Hankey, J. Sudweeks, and T. Dingus. Analysis of Lane-Change Crashes and Near-Crashes. National Technical Information Service, Springfield, VA 22161, 2009

[9] Shinar, D., Psychology on the road: The human factor in traffic safety. Wiley New York, (1978)

[10] Sun, D. J., \& Elefteriadou, L. (2011). Lane-changing behavior on urban streets: a focus group-based study. Applied ergonomics, 42(5), 682-91. doi:10.1016/j.apergo.2010.11.001.

[11] X. G. Li, B. Jia, Z. Y. Gao, and R. Jiang, "A realistic two-lane cellular automata traffic model considering aggressive lane- changing behavior of fast vehicle," PhysicaA, vol. 367, pp. 479-486, 2006.

[12] K. Nagel and M. Schreckenberg, "A cellular automaton model for freeway traffic," Journal of Physics I France, vol. 2, no. 12, pp.22212229, 1992.

[13] K. Nagel, Wolf, Wagner, and Simon, "Two-lane traffic rules for cellular automata: A systematic approach," Physical Review E, vol.58, no.2, 1998.

[14] S. Maerivoet and B. D. Moor, "Transportation Planning and Traffic Flow Models," 05-155, Katholieke Universiteit Leuven, Department of Electrical Engineering ESAT-SCD (SISTA), July 2005.

[15] M. Rickert, K. Nagel, M. Schreckenberg, and A. Latour, "Two Lane Traffic Simulations using Cellular Automata," vol. 4367, no. 95, 1995.

[16] W. Knospe, L. Santen, A. Schadschneider, and M. Schrekenberg, "Disorder effects in cellular automata for two lane traffic," Physica A, vol. 265, no. 3-4, pp. 614-633, 1998.

[17] A. Awazu, "Dynamics of two equivalent lanes traffic flow model: selforganization of the slow lane and fast lane," Journal of Physical Society of Japan, vol. 64, no. 4, pp. 1071- 1074, 1998.

[18] E. G. Campri and G. Levi, "A cellular automata model for highway traffic," The European Physica Journal B, vol. 17, no. 1, pp. 159-166, 2000.

[19] L. Wang, B. H. Wang, and B. Hu, "Cellular automaton traffic flow model between the Fukui-Ishibashi and Nagel- Schreckenberg models," Physical Review E, vol. 63, no. 5, Article ID 056117, 5 pages, 2001.

[20] B. Jia, R. Jiang, Q. S. Wu, and M. B. Hu, "Honk effect in the two-lane cellular automaton model for traffic flow," Physica A, vol. 348, pp. 544$552,2005$.

[21] D. Chowdhury, L. Santen, and A. Schadschneider, "Statistical physics of vehicular traffic and some related systems," Physics Report, vol. 329, no. 4-6, pp. 199-329, 2000.

[22] W. Knospe, L. Santen, A. Schadschneider, and M. Schreckenberg, "A realistic two-lane traffic model for highway traffic," Journal of Physics A, vol. 35, no. 15 , pp. 3369-3388, 2002.

[23] W. Knospe, L. Santen, A. Schadschneider, and M. Schreckenberg, "Empirical test for cellular automaton models of traffic flow," Phys. Rev. E, vol. 70, 2004.

[24] Nagatani, T., "Self Organization and Phase Transition in the Traffic Flow Model of a Two-Lane Roadway," Journal of Physics A, Vol. 26, pp. 781-787, 1993.

[25] Shahar, A., Van Loon, E., Clarke, D., \& Crundall, D. Attending overtaking cars and motorcycles through the mirrors before changing lanes. Accident; analysis and prevention, 44(1), 104-10, 2012. 
[26] Rayner, K., Warren, T., Juhasz, B.J., Liversedge, S.P. The effect of plausibility on eye movements in reading. Journal of Experimental Psychology: Learning, Memory and Cognition 30, 1290-1301, 2004.

[27] Benjaafar, S., \& Dooley, K. (1997). Cellular automata for traffic flow modeling. Minneapolis, MN, University of. Retrieved from http://ntl.bts.gov/lib/21000/21100/21189/PB99103996.pdf.

[28] Paz, A., \& Peeta, S. (2009). Information-based network control strategies consistent with estimated driver behavior. Transportation Research Part B: Methodological, 43(1), 73-96. doi:10.1016/j.trb.2008.06.007.

\section{AUTHORS PROFILE}

Kohei Arai He received BS, MS and PhD degrees in 1972, 1974 and 1982, respectively. He was with The Institute for Industrial Science and Technology of the University of Tokyo from April 1974 to December 1978 and also was with National Space Development Agency of Japan from January, 1979 to March, 1990. During from 1985 to 1987, he was with Canada Centre for Remote Sensing as a Post-Doctoral Fellow of National Science and Engineering Research Council of Canada. He moved to Saga University as a Professor in Department of Information Science on April 1990. He was a councilor for the Aeronautics and Space related to the Technology Committee of the Ministry of Science and Technology during from 1998 to 2000 . He was a councilor of Saga University for 2002 and 2003. He also was an executive councilor for the Remote Sensing Society of Japan for 2003 to 2005 . He is an Adjunct Professor of University of Arizona, USA since 1998. He also is Vice Chairman of the Commission-A of ICSU/COSPAR since 2008. He wrote 34 books and published more than 510 journal papers. He is Editor-in-Chief of IJACSA and International Journal of Intelligent Systems and Applications. http://teagis.ip.is.saga-u.ac.jp/bib.html. 